- Article •

\title{
The onset of the tetrabonded structure in liquid water
}

\author{
Carla Andreani ${ }^{1,2}$, Carmelo Corsaro ${ }^{3}$, Domenico Mallamace $^{3}$, \\ Giovanni Romanelli ${ }^{4 *}$, Roberto Senesi ${ }^{1,2}$, and Francesco Mallamace ${ }^{5,6}$ \\ ${ }^{1}$ Dipartimento di Fisica and NAST Center, Università degli Studi di Roma Tor Vergata, Rome 00133, Italy; \\ ${ }^{2}$ CNR-IPCF, Istituto per i Processi Chimico-Fisici del CNR di Messina, Messina 98158, Italy; \\ ${ }^{3}$ Dipartimento MIFT, Università di Messina, Messina 98166, Italy; \\ ${ }^{4}$ ISIS Facility, Rutherford Appleton Laboratory, Chilton, Didcot, Oxfordshire OX11 OQX, UK; \\ ${ }^{5}$ Istituto dei Sistemi Complessi (ISC) - CNR, Rome 00185, Italy; \\ ${ }^{6}$ Center for Polymer Studies and Department of Physics, Boston University, Boston 02215, USA
}

Received December 18, 2018; accepted April 3, 2019; published online May 27, 2019

\begin{abstract}
Water properties are dominated by the hydrogen bond interaction that gives rise in the stable liquid phase to the formation of a dynamical network. The latter drives the water thermodynamics and is at the origin of its well known anomalies. The HB structural geometry and its changes remain uncertain and still are challenging research subjects. A key question is the role and effects of the HB tetrahedral structure on the local arrangement of neighboring molecules in water. Here the hydrogen dynamics in bulk water is studied through the combined use of Neutron Compton Scattering and NMR techniques. Results are discussed in the framework of previous studies performed in a wide temperature range, in the liquid, solid, and amorphous states. For the first time this combined studies provide an experimental evidence of the onset of the water tetrahedral network at $T \sim 315 \mathrm{~K}$, originally proposed in previous studies of transport coefficients and thermodynamical data; below this temperature the local order in water changes and the lifetime of local hydrogen bond network becomes long enough to gradually develop the characteristic tetrahedral network of water.
\end{abstract}

water, neutron compton scattering, NMR

PACS number(s): $\quad 29.30 . \mathrm{Hs}, 82.56 . \mathrm{b}, 05.70 . \mathrm{Fh}$

Citation: C. Andreani, C. Corsaro, D. Mallamace, G. Romanelli, R. Senesi, and F. Mallamace, The onset of the tetrabonded structure in liquid water, Sci. China-Phys. Mech. Astron. 62, 107008 (2019), https://doi.org/10.1007/s11433-018-9408-2

\section{Introduction}

Water, essential to life, is still a challenging research subject $[1,2]$. If compared with other materials, water is, in the thermodynamical sense, a very complex system with many anomalies characterizing its phase diagram, particularly in the liquid phase [3]. Such anomalies are at the origin of the many open questions regarding both dynamical and structural water behaviors [4-9]. For these reasons, water has been, and still is extensively studied using

*Corresponding author (email: giovanni.romanelli@stfc.ac.uk) a variety of experimental and theoretical models [10-14]. Experiments range from classical collective thermodynamic techniques (such as dielectric constant or specific heat), to investigations probing the microscopic order (such as $\mathrm{X}$-ray and neutron diffraction and nuclear magnetic resonance (NMR) spectroscopy [15]), and from vibrational spectroscopy (such as Raman, infrared and inelastic X-ray [16-18] and neutron scattering) to the study of single-particle dynamics using neutron compton scattering (NCS) [19-22]. Despite these efforts, a thorough understanding of the structure of liquid water in the whole pressure-temperature $(P-T)$ diagram is 
still matter of debate within the scientific community.

Current results from experiments, simulations, and models converge on the fact that the intermolecular hydrogen bond (HB) interactions determine the chemical physics of bulk water and its solutions [23], and that the HB structure in liquid water is temperature dependent [2].

Upon cooling, the HB interaction in water orders the nearest neighbor molecules, that gradually assume the characteristic tetrahedral ice-like geometry: each water molecule having four nearest neighbors, a hydrogen receptor to two of them and a hydrogen donor from the other two. As opposed to the permanent four-coordinated network held together by HB in ice, the network in the liquid phase is local and transient. Areas of local tetrahedral order possess a larger specific volume than the overall average; the entropy, on the other hand, always decreases upon cooling because the specific heat is, of necessity, positive. Thus, as $T$ decreases, for the progressive increase in tetrahedral order, the local specific volume increases. Recent studies on the thermodynamical response functions suggest that the onset of these tetrahedral water "patches" occurs at $T^{*} \simeq 315 \mathrm{~K}$, whereas at higher temperatures the water behavior is the same of a simple liquid [24]. The situation is illustrated in Figure 1, where the thermal expansion coefficient, $\alpha_{P}(T, P)$, is shown as a function of temperature for several different pressures in the range 1 bar- 8 kbar.

Taking into account that $\alpha_{P}(T, P)$ represents the crosscorrelations of the volume $(V)$ and entropy $(S)$ fluctuations $\alpha_{P} \sim\langle\Delta S \Delta V\rangle$, one can note the existence of a universal temperature where all curves cross each other, i.e., a crossover for the water behavior from simple to anomalous liquid [24, 25]. In particular, in the high-temperature regime (right side of the picture with respect to $T^{*}$ ), water behaves as a normal liquid,

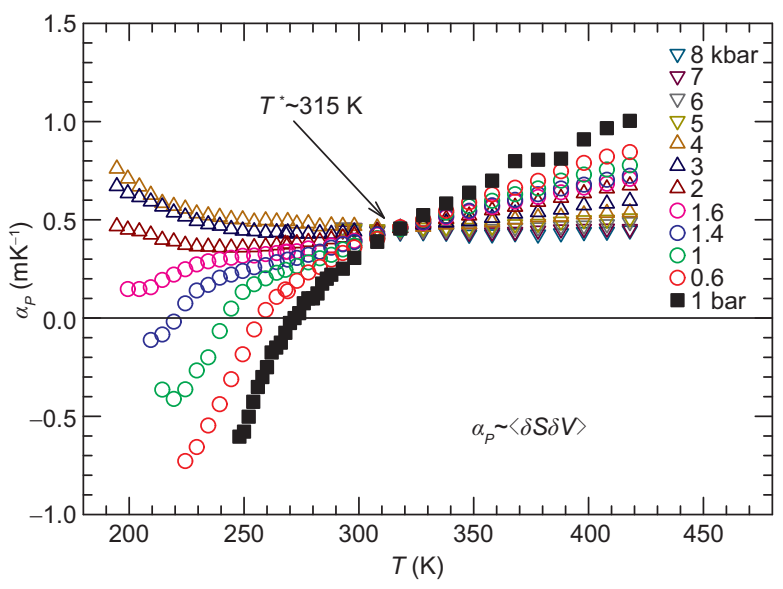

Figure 1 (Color online) The temperature dependence of the thermal expansion coefficient at several different pressures in the range 1 bar- 8 kbar. The universal expansivity point, where all curves cross, is well evident and indicated by the arrow. Figure adapted from refs. [24, 25]. whereas all water anomalies belong in the left side of the picture. An example can be found in the self-diffusion coefficient of water studied by Simpson and Carr [26] using NMR experiments. The latter shows that, at the same crossover temperature, a change in the behavior of the transport parameter occurs, from an Arrhenius at $T>315 \mathrm{~K}$ to a superArrhenius at $T<315 \mathrm{~K}$, accompanied by the violation of the Stokes-Einstein relation.

In a recent work, from $\alpha_{P}(T, P)$ cross-correlations, $K_{T}(T, P)$ isothermal compressibility, and NMR self-diffusion coefficients, it has been suggested that $T^{*}$ represents the onset of the HB network, thus of the water anomalous behavior in the supercooled regime, such as the negative value in $\alpha_{P}(T, P)$. This suggests that in water two "phases" coexist, one of which is composed by the transient HB network and the other by the remaining molecules, i.e., water is polymorphic for $T<T^{*}$ [27], a situation like the one experimentally observed in amorphous ice [28-30]. Ice polymorphism is at the origin of special scenarios proposed to reproduce the chemical and physical properties of water, and especially to explain the thermodynamics of the liquid phase based on the HB interaction [27,31]. Currently, three are the hypotheses of main interest: the stability limit conjecture (SLC), the singularity-free scenario (SF), and the liquid-liquid phase transition (LLPT). The latter two assume that water properties are the result of HBs creating local transient structures composed of many molecules, increasing in number as temperature decreases [2]. These local "patches", or HB sub-domains [32,33], enhance the thermodynamical fluctuations (specific volume and entropy and their negative crosscorrelations) with anomalies that closely resemble those observed experimentally. The two observed amorphous states are the corresponding vitreous forms of low-density liquid (LDL) and high-density liquid (HDL) water. The difference between the SF and the LLPT is that, upon supercooling, the response functions increase sharply yet remaining finite in the first case, as opposed to a transition with critical fluctuations in the case of LLPT. The latter model also defines a special locus in the $P-T$ plane, the Widom line, where the HB network is fully realized, with the LDL phase dominating the HDL, and the water thermodynamics is characterized by maxima in the response-function fluctuations. The corresponding temperature is referred to as $T_{L}$, and at ambient pressure $T_{L} \simeq 225 \mathrm{~K}$ [34]. Such a picture is confirmed by experiments in confined water and simulations in the bulk [35-38]. By decreasing $T$ (and increasing $P$ ) the Widom line converges to a critical point (the liquid-liquid critical point, LLCP) where fluctuations diverge and the LDL phase dominates the liquid structure. In this context, the description of the $T$-dependent structure of water in terms of the HB properties (life-time, strength, length and angular distri- 
butions) represents an interesting challenge.

This paper presents a combined NCS and NMR study of the local order in water in a broad temperature interval from the critical region to the deep supercooled phase. New NCS measurements have been performed in a $T$ range around $T^{*} \simeq 315 \mathrm{~K}$. NCS, also known as deep inelastic neutron scattering (DINS), is a unique technique to measure the ground-state dynamics of atomic nuclei [39], routinely used to derive the hydrogen momentum distribution, $n(p)$, and hydrogen mean kinetic energy, $\left\langle E_{\mathrm{K}}\right\rangle$ in liquid water [40] and ice $[41,42]$, heavy water [43], and water confined in porous materials $[44,45]$. These physical quantities provide information on the local environment of the $\mathrm{H}$ nucleus in the $\mathrm{HB}$ network [46]. NCS operates in a scattering regime of high momentum, $\hbar q$, and energy, $\hbar \omega$, transfers i.e., $\hbar q$ up to hundreds of $\AA^{-1}$ and $\hbar \omega$ up to hundreds of eV [39]. As far as NMR is concerned, the magnetic shielding tensor $\sigma$ measured by this technique relates the applied magnetic field to the local field experienced by the magnetic moment of the studied hydrogen proton (i.e., ${ }^{1} \mathrm{H}$ NMR). The sensitivity of the shielding tensor to the local electronic environment makes NMR a powerful probe of the local environment [47-49]. Results from NMR and NCS complement those obtained from the diffraction studies. Both NMR and NCS provide massresolved experimental spectra. In the case of NCS, individual nuclei appear as independent peaks Doppler broadened by the motion of the nucleus in the surrounding local potential due to nuclear zero-point and thermal energies [39].

By combining results from present NCS and NMR measurements with those obtained in previous investigations, we have been able to mirror the water structural changes, and show the onset of the HB tetrahedral structure as well as its growth by decreasing temperature. The onset occurs around $T^{*} \simeq 315 \mathrm{~K}$. These results show the thermal evolution of the local dynamics of HB "patches" in water, confirming the existence of two states in liquid water inside the stable phase.

\section{Methods}

\subsection{Neutron compton scattering}

NCS measurements on water around $T^{*} \simeq 315 \mathrm{~K}$ were performed to determine the hydrogen $n(p)$ and mean kinetic en$\operatorname{ergy}\left\langle E_{K}\right\rangle$ [50]. NCS measurements were carried out on the VESUVIO beamline at the ISIS pulsed neutron and muon source (Rutherford Appleton Laboratory, Chilton, Didcot, UK) [51,52]. Liquid water was contained in a flat aluminium container with sample thickness of $0.5 \mathrm{~mm}$ and inner container surfaces coated with a 0.01-mm-thick Polytetrafluoroethylene (PTFE) layer. NCS measurements were performed at four temperatures, 300, 315, 320, $325 \mathrm{~K}$ and at atmospheric pressure. Temperature was controlled with the precision of $0.1 \mathrm{~K}$. Details of the experimental apparatus and measurement technique are reported in previous papers $[22,53,54]$. NCS is described within the framework of the impulse approximation (IA) [20,55], valid at high energy and momentum transfers $[21,56,57]$, with the scattering intensities centred along the atomic recoil lines [21]. In the present experiment, the momentum and energy transfer were in the ranges $27 \AA^{-1}<\hbar q<230 \AA^{-1}$ and $2.5 \mathrm{eV}<\hbar \omega<800 \mathrm{eV}$, respectively. From each $l$-th detector, we obtained a hydrogen neutron compton profile, $F_{l}(y, q)$. These functions represent the hydrogen longitudinal momentum distribution along the direction of $q$. Full details on the NCS formalism, the experimental set up, measurements, and data analysis are reported in the Supporting information online. The average of individual $F_{l}(y, q)$ functions, namely $F(\widetilde{y})$, is a useful quantity to appreciate the quality of the data and fits. For each sample, a global fit over individual spectra was accomplished using a parametric model for the $n(p)$, i.e., a three dimensional anisotropic Gaussian line-shape derived from a quasiharmonic model [58]. The latter has been employed in previous NCS investigations to reveal the local environment of hydrogen in polycrystalline ice [42,54], amorphous ice [59] and heavy water [43]. Within this framework, the experimental $n(p)$ of an amorphous sample, such as a liquid, can be modelled by spherically-averaging a multivariate Gaussian function according to the following equation [42]:

$4 \pi p^{2} n(\mathbf{p})=\left\langle\frac{\delta(p-|\mathbf{p}|)}{\sqrt{8 \pi^{3}} \sigma_{x} \sigma_{y} \sigma_{z}} \exp \left(-\frac{p_{x}^{2}}{2 \sigma_{x}^{2}}-\frac{p_{y}^{2}}{2 \sigma_{y}^{2}}-\frac{p_{z}^{2}}{2 \sigma_{z}^{2}}\right)\right\rangle$

The set of parameters $\sigma_{\alpha}(\alpha=x, y, z)$, determines the anisotropy in the momentum distribution, with the $z$ direction usually assumed along the direction of the $\mathrm{OH}$ covalent bond, and $x$ and $y$ in the perpendicular plane.

In the case of a nucleus in a harmonic and isotropic potential, $n(\mathbf{p})$ depends only on the momentum modulus $p=$ $\left|\left(p_{x}, p_{y}, p_{z}\right)\right|$, and it can be expressed as an univariate Gaussian function of the form:

$n(p)=\left(1 / \sqrt{2 \pi} \sigma_{\mathrm{NCP}}\right) \exp \left(-p^{2} / 2 \sigma_{\mathrm{NCP}}^{2}\right)$,

where $\sigma_{\mathrm{NCP}}^{2}=\sum_{\alpha} \sigma_{\alpha}^{2} / 3$ [39]. In these terms, $\sigma_{\mathrm{NCP}}$ is related to the ground-state nuclear mean kinetic energy as: $\left\langle E_{\mathrm{K}}\right\rangle=3 \hbar^{2} \sigma_{\mathrm{NCP}}^{2} / 2 M$. We notice that in a liquid or amorphous material molecules are randomly oriented with respect to the laboratory reference of frame, thus requiring the angular average in eq. (1). However, the average of a multivariate Gaussian function still differs from a univariate Gaussian 
function of width $\sigma_{\mathrm{NCP}}$. The difference between the two functions can be generally expressed as a series of Hermite polynomials with each coefficient being a function of $\sigma_{\alpha}$ [53]. Therefore, the local anisotropy in the local potential affecting hydrogen is an accessible observable in DINS experiments even for liquid or amorphous samples.

\subsection{Nuclear magnetic resonance}

NMR experiments were performed at ambient pressure in the temperature interval $265 \mathrm{~K}<T<335 \mathrm{~K}$ (with steps of $1 \mathrm{~K}$ ) using a Bruker AVANCE NMR spectrometer (Germany) operating at $700 \mathrm{MHz}$ proton resonance frequency. The temperature was controlled with a stability of $0.05 \mathrm{~K}$. We measured the chemical shift $\widehat{\delta}$ according to standard procedures described elsewhere [60]. In addition, literature data for ice and water were also considered. The experiment was performed on bulk water in a thermodynamic region previously explored with apparati of different sensitivity, providing a new set of data with denser $T$ sampling and better resolution [61-63].

In NMR experiments, one measures the chemical shift $\widehat{\delta}$ as the change of resonance frequency of a nucleus with respect to a given standard, and the magnetic shielding tensor $\widehat{\sigma}^{\text {NMR }}$ is then obtained from the relation:

$\widehat{\delta}=\mathbf{1} \sigma_{\text {iso }}^{\mathrm{NMR}}-\widehat{\sigma}^{\mathrm{NMR}}$,

where $\mathbf{1}$ is the unit matrix and $\sigma_{\text {iso }}^{\mathrm{NMR}}$ is the isotropic value or trace $\left(\sigma_{\text {iso }}^{\mathrm{NMR}} \equiv \operatorname{Tr}\left(\widehat{\sigma}^{\mathrm{NMR}} / 3\right)\right)$ of the shielding tensor of the standard reference used in the NMR experiments. It is assumed that $\delta$ corresponds to a linear response of the electronic structure of the system under investigation to an external magnetic field $B_{0}$, as $B(j)=\left(1-\delta_{j}\right) B_{0}$, where $j$ is an index identifying the chemical environment $[47,48]$. Both $\widehat{\delta}$ and $\widehat{\sigma}^{\text {NMR }}$ can be measured by the free induction decay (FID) that contains information about the set of all nuclear species in the studied sample whose resonance frequencies lie within the harmonic content of the NMR radio frequency (RF) pulse [63].

For a molecular liquid (like water) $\widehat{\sigma}^{\mathrm{NMR}}$ is nearly axially symmetric, and it is obtained by the isotropic average $\sigma_{\text {iso }}^{\mathrm{NMR}}$ and the anisotropy $\Delta \sigma^{\mathrm{NMR}}=\sigma_{33}^{\mathrm{NMR}}-\left(\sigma_{11}^{\mathrm{NMR}}+\sigma_{22}^{\mathrm{NMR}}\right) / 2$ $\left(\sigma_{11}^{\mathrm{NMR}}, \sigma_{22}^{\mathrm{NMR}}\right.$, and $\sigma_{33}^{\mathrm{NMR}}$ are the three principal components of $\left.\widehat{\sigma}^{\mathrm{NMR}}\right)$. $\sigma_{\text {iso }}^{\mathrm{NMR}}$ is given by the measured proton chemical shift relative to a reference state through the relation $[64,65]$

$\delta=\sigma_{\text {iso }}^{\mathrm{NMR}, \mathrm{ref}}-\sigma_{\text {iso }}^{\mathrm{NMR}}+\left(A-\frac{1}{3}\right)\left(\chi^{\mathrm{ref}}-\chi\right)$,

where $\chi$ is the magnetic susceptibility and $A$ depends on the sample shape and orientation (for a sphere $A=1 / 3$ ). If the isotropic shielding reference value, $\sigma_{\text {iso }}^{\text {NMR,ref }}$, is obtained in an absolute scale, the value of $\sigma_{\text {iso }}^{\mathrm{NMR}}$ can be directly obtained by the measured chemical shift [63].

The shielding tensor was used for the ice HB structure [65]; and in the stable liquid phase and near its critical point (and thus $\sigma_{\text {iso }}^{\mathrm{NMR}}$ ) it is affected by the HB depletion of the electron density around the proton $[15,66]$, whereas the induced magnetic field by the acceptor oxygen electrons perturbs the anisotropy [67]. This stresses the primary role of $\mathrm{HB}$ and of the corresponding tetrahedral network in water systems. More precisely, the HB geometry can be determined by combining proton $\widehat{\sigma}^{\text {NMR }}$ measurements and ab initio density functional theory (DFT) calculations. In such a way the moments of the distributions of HB length $\left(R_{H O}\right)$ and angle $(\beta)$ measures the thermal disorder of the HB network, whereas the chemical shift is related to the average $\mathrm{HB}$ number [15,61].

For water in the stable liquid phase, the fast molecular tumbling isotropically averages its shielding anisotropy. Well different is, instead, the situation in the deep supercooled regime and for amorphous water (LDA and HDA) for the effects of the growing $\mathrm{HB}$ network. While in the liquid water stable phase the HB life-time is of the order of some picoseconds, on going toward the amorphous phase via the supercooling, such a time grows of many orders of magnitude by increasing not only the HB network stability but also its size. In such a situation a certain influence in the NMR tensors ( $\delta$ and $\sigma$ ) is detectable. These structural effects can be determined as asymmetries in the NMR line-shapes or from its contribution to the proton spin relaxation rate (see e.g., ref. [66]).

\section{Results and discussion}

Despite the different radiation-matter interaction underpinning compton scattering (CS) and NMR, the scattering matrix involved in the process is essentially the same. Hence, one can assume that both the shielding tensor and the momentum distribution mirror the same anisotropy of the $\mathrm{H}$ potential [18]. In general terms, CS techniques are unique probes of the ground-state momentum density of electrons (X-ray CS, XCS) or nuclei (NCS) in materials [17]. Both XCS and NCS, when benchmarked with computer simulations [18, 42, 54, 68], have emerged as important techniques to interpret the local molecular structure of water and the effects of changes in the HB network on the local coordination. As far as the electron momentum density is concerned, a study of liquid water based on NMR and XCS was presented in ref. [18]. The XCS measurements were performed at $T=273.5,277,288,298,323$, and $363 \mathrm{~K}$, with data at $298 \mathrm{~K}$ used as a reference (an analogous study was also reported from $\mathrm{D}_{2} \mathrm{O}$ [69]). In that work, changes in the $\mathrm{X}$ - 
ray compton profile (XCP) were observed upon increasing $T$ within the stable liquid phase, and related to the HB weakening. By combining DFT and the NMR data on the distribution function of the HB geometries [66], the XCPs have been related to the angle and $\mathrm{OH}$ distance of HBs. Results from the XCS study of liquid water [18] showed significant difference for $T$ above $320 \mathrm{~K}$ with respect to $T$ below $320 \mathrm{~K}$. In particular, XCS results showed a change in the HB behaviors at $T<$ $320 \mathrm{~K}$, for $q \simeq 1$ and $q \simeq 1.8$ (atomic units) where the XCPs are sensitive to the correlation between the intramolecular bond length and the HB geometry [18,69].

One should notice that $\mathrm{CS}$ is sensitive to the local $\mathrm{HB}$ structure, as it involves high values of the momentum transfer, whereas NMR provides a spatial average over the HB network for, in this case, $q \simeq 0$. To enforce this point, we show in the following discussion how NCS data are sensitive to a clear $\mathrm{HB}$ rearrangement near $T^{*}$, and by comparison to NMR results in the same temperature range, they support the idea of the onset of the local tetrabonded structure in liquid water.

Figure 2 shows the hydrogen chemical shift in liquid water, $\delta$, as a function of the temperature from the critical region to the deep supercooled regime $[61,63]$. Data in the case of water confined in emulsions [70] and in nanotubes [60] are also considered. The enlarged region around $315 \mathrm{~K}$, shown in the inset, allows a more precise observation of the change in the chemical shift strictly related with that in the local order of

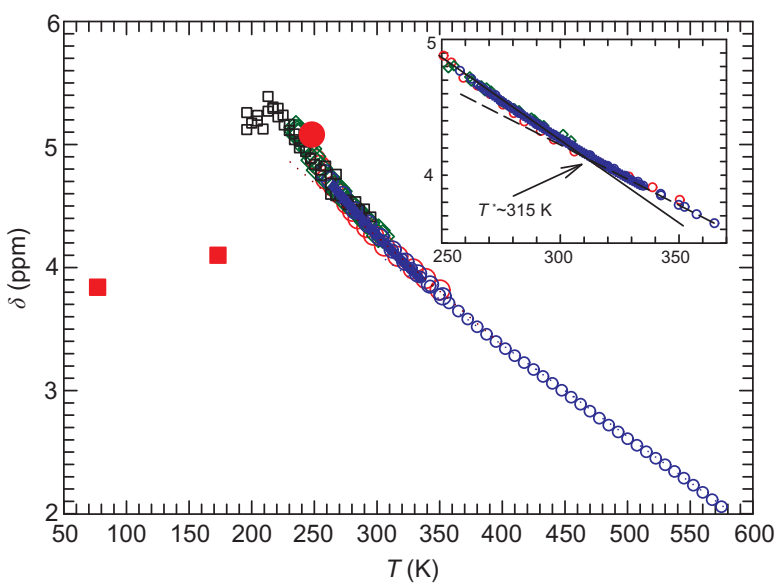

Figure 2 (Color online) The water chemical shift, $\delta$, measured in bulk water (blue triangles, actual data), from the critical region to the deep supercooled regime (blue circles) [61,63]. In the latter regime, the corresponding values of $\delta$ are measured for water inside large capillary (empty red circles), and emulsions with radius of the order of $10 \mu \mathrm{m}$ (green diamonds) [70]. The $\delta(T)$ chemical shift data of water confined in nanotubes of the $\AA$ size (black squares) [60] and ice (red circle) [71], polycrystalline ice (red square) [62] are also reported. The dotted red and blue lines are the data fitting in the regions 330-370 K and 390-580 K, respectively. The inset shows a zoomed region around $315 \mathrm{~K}$ in order to highlight the changes of the local order reflected in the change of the NMR chemical shift. the system. It can be observed that the $\delta(T)$ chemical shift increases by decreasing $T$, up to a maximum at about $T \sim$ $220 \mathrm{~K}$, then decreases down to the values of polycrystalline ice (large red squares). The several studies carried out on $\delta(T)$ suggest that this physical quantity provides a direct measurement of the number of HBs in water. Thus, from Figure 2, near the critical region and in the gas phase one observes only water monomers and dimers, whereas in the liquid phase at ambient condition water trimers and tetramers are formed [46,61,63]; the slope of the $\delta(T)$ chemical shift crossover at about $370 \mathrm{~K}$ marks the distinction between these two regions.

Figure 3 shows the experimental averaged $F(\widetilde{y})$ for $\mathrm{H}$ as a function of the $y$ (black circle) at $300 \mathrm{~K}$ from our new NCS measurements, and its best fit (continuous line); the insert shows the radial momentum distribution, $4 \pi p^{2} n(p)$, as a function of $p[21,22,53]$, with $\sigma_{\mathrm{NCP}}$ being the variance. In the case of water at $T=300 \mathrm{~K}$, the resulting parameters obtained from the fit using eq. (2) are: $\sigma_{\mathrm{NCP}}=(4.85 \pm 0.03) \AA^{-1}$ and $\left\langle E_{\mathrm{K}}\right\rangle=(146 \pm 2) \mathrm{meV}$, and additional details on the fitting procedure and results are provided in the Supporting information. Results of similar statistical accuracy have been obtained for the NCS data sets at the other temperatures of the experiment.

Figure 4 shows the $\mathrm{H}$ mean kinetic energy $\left\langle E_{\mathrm{K}}\right\rangle$ obtained from the present and previous experiments at different temperatures in the liquid and supercritical phases together with several forms of ices. In particular, the figure reports NCS data from: ice Ih at $T=5,71$ [54] and $271 \mathrm{~K}$ [72]; amorphous ices at $T=80 \mathrm{~K}$ [59]; and liquid and supercritical water at $T$ $=300,423,523,573$, and $673 \mathrm{~K}$ [73]. We observe that the $\left\langle E_{\mathrm{K}}\right\rangle$ value decreases from $\left\langle E_{\mathrm{K}}\right\rangle=178 \mathrm{meV}$, in the supercritical region at $T=673 \mathrm{~K}$, down to about $\left\langle E_{\mathrm{K}}\right\rangle=148 \mathrm{meV}$, at $T=315 \mathrm{~K}$, then significantly increasing to $\left\langle E_{\mathrm{K}}\right\rangle=155 \mathrm{meV}$

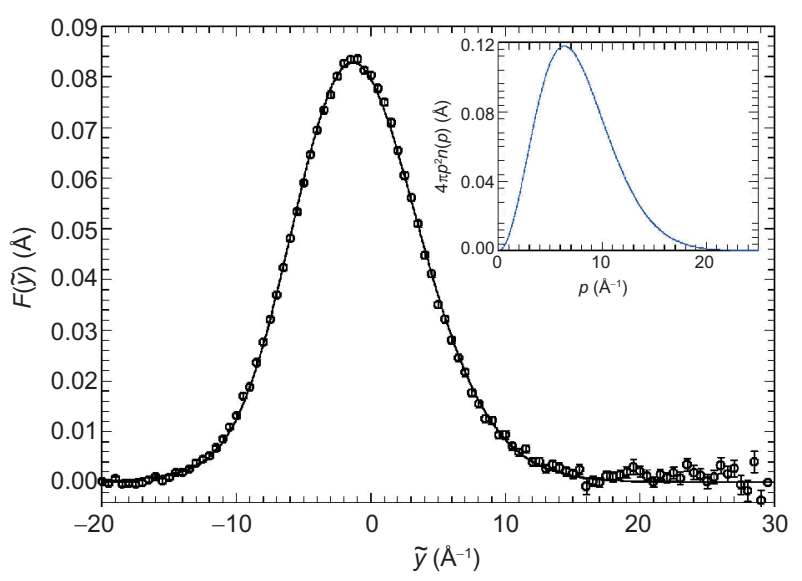

Figure 3 (Color online) The $F(\widetilde{y})$ function for $H$ at $300 \mathrm{~K}$ (black circles) and the best fit (continuous line). The insert reports the $4 \pi p^{2} n(p)$ function versus the momentum $p$ (see text) (blue line). 
when crossing the melting temperature, finally slowly decreasing at lower temperatures. The main contribution to the nuclear ground-state energy arises from the water vibrational modes [22], that are sensitive to both the intramolecular structure and geometry, as well as to the changes of the local HB network. We interpret the increase in kinetic energy at $T \sim 315 \mathrm{~K}$ as due to the change of the molecular order, and particularly to the onset of the tetrahedral network at this temperature. To the best of our knowledge, so far this behavior of NCS results has been observed for liquid water only, whereas in other liquids $\left\langle E_{\mathrm{K}}\right\rangle$ increases monotonically as temperature increases. Therefore, for the first time NCS studies provide an experimental evidence of the onset of the water tetrahedral network at $T^{*} \sim 315 \mathrm{~K}$.

Refs. [74, 75] report X-ray and neutron diffraction results on water and ice over a wide temperature range. One can notice that the significant changes in the O-O pair distribution function between crystalline ice, amorphous ices, and liquid water are all well mapped onto measurable changes in the hydrogen $\left\langle E_{K}\right\rangle$ in Figure 4 . On the other hand, the same refs. [74, 75] show no significant change in the diffraction observables in liquid water across $T^{*}$. Such discrepancy with our NCS results can be explained by considering that a quickly changing HB structure can be captured in NCS experiments, where the high-energy transfer allows the probing of the system on the fs time scale. The same HB structural rearrangement can elude diffraction techniques, where the time scale probed coincides with the time of the measurement. Moreover, it has been recently pointed out $[78,79]$

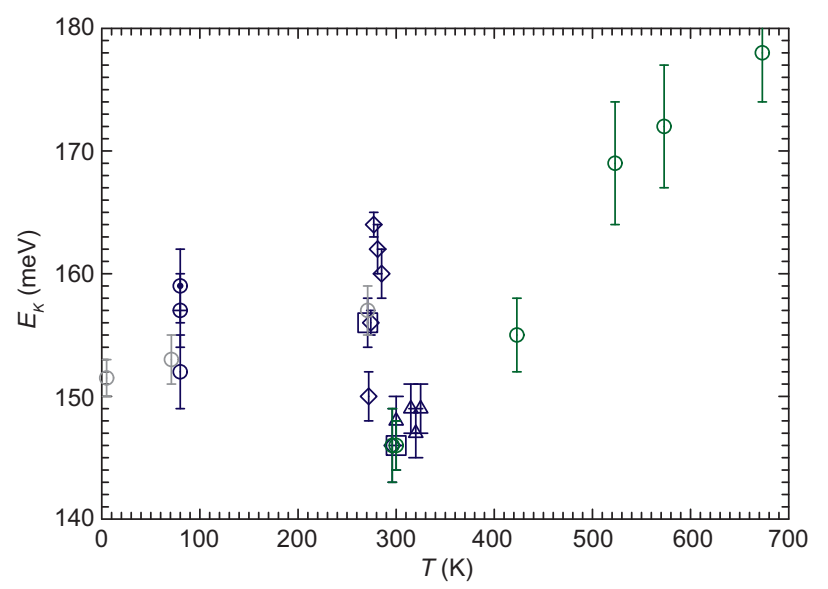

Figure 4 (Color online) The $\mathrm{H}$ mean kinetic energy $\left\langle E_{\mathrm{K}}\right\rangle$ obtained from the NCP measured at different temperatures in the liquid and supercritical phases together with several types of ices: ice Ih (grey circles) at $T=5,71$ [54] and $271 \mathrm{~K}$ [72]; amorphous ice (dark-blue circles: low density as empty circle; high density as crossed circle; and very high density as dotted circle) at $80 \mathrm{~K}$ [59]; and water in the stable liquid and supercritical phases (dark green circles) at $T=300,423,523,573$ and $673 \mathrm{~K}$ [73]. Dark-blue diamonds refer to data around density maximum [76]; dark blue squares at 271 and $300 \mathrm{~K}$ from ref. [77]; and dark-blue triangles refer to the present data. how a certain level of discrepancy on the measured value of the $\mathrm{O}-\mathrm{O}$ distance still remains in the literature.

The idea of the temperature $T^{*}$ was originally proposed in previous studies of the transport coefficient and other thermodynamic data [24,25], where it was suggested that $T^{*}$ was at the origin of the anomalous behavior of liquid water and the coexistence of two distinct liquids in water. In fact, by considering old and new data of both the isothermal compressibility $K_{T}(T, P)$ and the coefficient of thermal expansion $\alpha_{P}(T, P)$, it was observed that $K_{T}(T, P)$ showed a minimum at $T^{*} \simeq(315 \pm 5) \mathrm{K}$ for all the investigated pressures. Moreover, from the $\alpha_{P}(T, P)$ behavior, it was observed that the curves at different $P$, crossed at the same $T^{*}$. In other words, these data show a "singular and universal expansivity point" at $T^{*}$ and $\alpha_{P}\left(T^{*}\right) \simeq 0.44 \times 10^{-3} \mathrm{~K}^{-1}$ (Figure 1). This temperature, unlike other water singularities, is thermodynamically consistent, as one can consider the relationship connecting the two thermodynamic response functions [25].

Although NCS and NMR do not provide a direct measurement of the HB length and angle (and their correlations) as a function of the thermodynamic variables, the two techniques are sensitive to water's anisotropy through $\sigma_{\mathrm{NCP}}$ and $\sigma_{\text {iso }}^{\mathrm{NMR}}$, respectively. As mentioned, NMR averages the local order and anisotropy on a more large length scale (mesoscopic) than the NCS, whereas the latter investigates the local potential at the atomic scale. Such a difference becomes apparent when looking at Figure 5, where we plot both $\sigma_{\mathrm{NCP}}(T)$ and $\sigma_{\text {iso }}^{\mathrm{NMR}}(T)$ as functions of temperature. These quantities are plotted on two different vertical scales: on the left side $\sigma_{\mathrm{NCP}}(T)$ is expressed in $\AA^{-1}$, and on the right side $\sigma_{\text {iso }}^{\mathrm{NMR}}(T)$ in ppm. Apart for an overall multiplicative factor, their thermal evolution is the same in the range $320 \mathrm{~K}<T<600 \mathrm{~K}$ within the experimental error bars: $\sigma_{\mathrm{NCP}}$ and $\sigma_{\text {iso }}^{\mathrm{NMR}}$ decrease

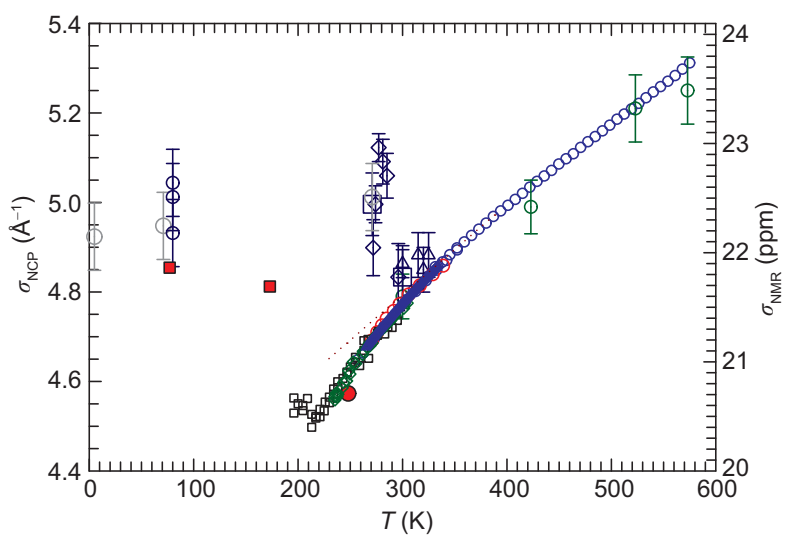

Figure 5 (Color online) The temperature behaviour of $\sigma_{\mathrm{NCP}}(T)$ (left scale) and $\sigma_{\text {iso }}^{\mathrm{NMR}}(T)$ (right scale). $\sigma_{\text {iso }}^{\mathrm{NMR}}$ is obtained from the chemical shift data reported in Figure 2 whereas the NCP data are obtained from the mean kinetic energy $\left\langle E_{\mathrm{K}}(T)\right\rangle$ reported in Figure 4. We have used the same symbols of Figures 2 and 4. 
practically at about the same rate as $T$ decreases. On the other hand, a marked difference is observed in the low temperature regime: on decreasing temperature, $\sigma_{\text {iso }}^{\mathrm{NMR}}$ decreases with continuity well inside the supercooled regime with a minimum at $T \sim 220 \mathrm{~K}$; on the contrary, $\sigma_{\mathrm{NCP}}$ shows a sharp increase below $T^{*}$, then again in the proximity of the density maximum at $T \sim 277 \mathrm{~K}$.

The differences observed between $\sigma_{\mathrm{NCP}}(T)$ and $\sigma_{\text {iso }}^{\mathrm{NMR}}(T)$ can be readily explained by considering the different scattering wave-vectors corresponding to the two techniques. On the one hand, NMR studies rely on values of the momentum transfer $q \equiv 0$, thus probing the response of the system over an averaged macroscopic spatial scale. On the other hand, the formalism of NCS, i.e., the IA, assumes $q \rightarrow \infty$, experimentally achieved with $q$ values around tens to hundreds of $\AA^{-1}$, over spatial scales of the fraction of the $\mathrm{OH}$ distance. Moreover, NCS also relies on large values of energy transfers, probing the system over the fs time scale. In this framework, it is possible to rationalize the different behavior of $\sigma_{\mathrm{NCP}}(T)$ and $\sigma_{\text {iso }}^{\mathrm{NMR}}(T)$ by the onset of phenomena that are both transient and very localised, and taking place at $T^{*} \simeq 315 \mathrm{~K}$ and lower temperatures. While NCS probes the single-particle dynamics, we suggest that the change in value of $\left\langle E_{K}\right\rangle$ also reflects structural ordering of the system, through the dependence of the vibrational modes on the molecular geometry, as well as the change in shape of the nuclear momentum distribution, also mirroring the effect of intramolecular interactions.

Thus, we identify $T^{*}$ as the temperature marking the onset of water $\mathrm{HB}$ tetrahedral structure or, more precisely, the LDL phase, that increases in size and stability on decreasing the temperature, and it is at the basis of the water anomalies. The observed $T$-behaviour of $\sigma_{\mathrm{NCP}}(T)$ reflects an increase in the correlation length of the structural fluctuations, confirming the suggestion of previous experimental and theoretical studies $[11,80,81]$. It must also be stressed that, assuming the existence of the two liquid phases, at temperatures about $T^{*}$ the HDL molecules dominate in number those of the LDL phase, whose number increases only by decreasing $T$. At lower temperatures, their population fraction is inverted [36], corresponding to the increase in the cooperative or tetrahedral character of HB, observed by experiments and numerical calculations $[11,82]$ at temperatures near the Widom line.

On the other hand, the NMR probe, working on macroscopic lengths, is insensitive to small sizes of the HB network, yet it reflects the thermal evolution of the averaged water HB length and angular distributions. Moreover, when the LDL water network dominates the HDL phase, and its characteristic size increases up to the mesoscopic size, the NMR sensitivity augments, presenting the observed minimum at $T \simeq 220 \mathrm{~K}$. At this temperature, the $\mathrm{HB}$ network is fully realized, with the LDL phase dominating over the HDL one, and the water thermodynamics is characterized by maxima in the response functions fluctuations.

\section{Conclusions}

The local order of liquid water at different temperatures, from the supercooled regime to the superheated ones, and in different forms of ice and glasses has been discussed in the framework of NCS and NMR measurements. In particular, the standard deviation of the nuclear momentum distribution, $\sigma_{\mathrm{NCP}}$ from NCS data, and the isotropic part of the magnetic shielding tensor $\sigma_{\text {iso }}^{\mathrm{NMR}}(T)$ from NMR experiments, have been presented as providing information about the same underlying anisotropy of the system. While NCS, probing the system over spatial scales of the order of the $\AA$ and timescales of the order of fs, is a sensitive probe of the local potential affecting hydrogen, NMR, working at momentum transfers $\mathbf{q}=0$, provides structural and dynamical details averaged over larger scales. Despite the two spectroscopic techniques do not probe directly the structure of the system under study, previous investigations have proved that they are sensitive to the rearrangement of the hydrogen-bonding network in water. In this framework, the data discussed in this work provide evidence of the onset of a local and transient tetrabonded HB network at $T^{*} \simeq 315 \mathrm{~K}$ and at lower temperatures. Below $T^{*}$ the $\mathrm{HB}$ lifetime becomes long enough to gradually develop the characteristic tetrahedral network of water. Our interpretation of the temperature dependence of NMR and NCS data in the deeply supercooled region supports the idea of the coexistence of LDL and HDL water phases at $T<T^{*}$, and that the size of the HB network increasing to mesoscopic scales for temperatures decreasing down to $\simeq 225 \mathrm{~K}$, near the Widom line, where LDL water dominates over the HDL phase.

This work was supported by the framework of past and present (2014-2020) agreements between the CNR and the STFC for collaborative research between Italy and ISIS.

\section{Supporting Information}

The supporting information is available online at phys.scichina.com and http://link.springer.com/journal/11433. The supporting materials are published as submitted, without typesetting or editing. The responsibility for scientific accuracy and content remains entirely with the authors.

1 H. E. Stanley, Liquid Polymorphism (Wiley, New York, 2013).

2 P. G. Debenedetti, and H. E. Stanley, Phys. Today 56, 40 (2003).

3 P. Ball, Chem. Rev. 108, 74 (2008).

4 P. G. Debenedetti, Metastable Liquids (Princeton University Press, Princeton, 1997), p. 407.

5 F. Mallamace, P. Baglioni, C. Corsaro, J. Spooren, H. E. Stanley, and S.-H. Chen, Riv. Nuovo Cimento 344, 253 (2011). 
6 F. Mallamace, C. Corsaro, D. Mallamace, P. Baglioni, H. E. Stanley, and S. H. Chen, J. Phys. Chem. B 115, 14280 (2011).

7 F. Mallamace, C. Corsaro, S.-H. Chen, and H. E. Stanley, Liquid Polymorphism (John Wiley \& Sons, Inc., Hoboken, 2013), pp. 203-262.

8 F. Mallamace, C. Corsaro, D. Mallamace, S.-H. Chen, and H. E. Stanley, Liquid Polymorphism (John Wiley \& Sons, Inc., Hoboken, 2013), pp. 263-308.

9 P. Kumar, and H. E. Stanley, J. Phys. Chem. B 115, 14269 (2011).

10 F. Perakis, K. Amann-Winkel, F. Lehmkühler, M. Sprung, D. Mariedahl, J. A. Sellberg, H. Pathak, A. Späh, F. Cavalca, D. Schlesinger, A. Ricci, A. Jain, B. Massani, F. Aubree, C. J. Benmore, T. Loerting, G. Grübel, L. G. M. Pettersson, and A. Nilsson, Proc. Natl. Acad. Sci. USA 114, 8193 (2017).

11 V. Bianco, and G. Franzese, Sci. Rep. 4, 4440 (2015).

12 P. H. Poole, I. Saika-Voivod, and F. Sciortino, J. Phys.-Condens. Matter 17, L431 (2005).

13 V. Holten, J. C. Palmer, P. H. Poole, P. G. Debenedetti, and M. A. Anisimov, J. Chem. Phys. 140, 104502 (2014), arXiv: 1312.4871.

14 J. L. F. Abascal, and C. Vega, J. Chem. Phys. 133, 234502 (2010).

15 K. Modig, B. G. Pfrommer, and B. Halle, Phys. Rev. Lett. 90, 075502 (2003).

16 M. J. Cooper, P. E. Mijnarends, N. Shiotani, N. Sakai, and A. Bansil, $X$-Ray Compton Scattering (Oxford University Press, Oxford, 2004).

17 M. J. Cooper, Rep. Prog. Phys. 48, 415 (1985).

18 M. Hakala, K. Nygärd, S. Manninen, S. Huotari, T. Buslaps, A. Nilsson, L. G. M. Pettersson, and K. Hämäläinen, J. Chem. Phys. 125, 084504 (2006).

19 G. I. Watson, J. Phys.-Condens. Matter 8, 5955 (1996).

20 J. M. F. Gunn, C. Andreani, and J. Mayers, J. Phys. C-Solid State Phys. 19, L835 (1986).

21 C. Andreani, D. Colognesi, J. Mayers, G. F. Reiter, and R. Senesi, Adv. Phys. 54, 377 (2005).

22 C. Andreani, M. Krzystyniak, G. Romanelli, R. Senesi, and F. Fernandez-Alonso, Adv. Phys. 66, 1 (2017)

23 D. Eisenberg, and W. Kauzmann, The Structure and Properties of Water (Oxford University Press, New York, 1969).

24 F. Mallamace, C. Corsaro, and H. E. Stanley, Sci. Rep. 2, 993 (2012).

25 F. Mallamace, C. Corsaro, D. Mallamace, C. Vasi, and H. E. Stanley, Faraday Discuss. 167, 95 (2014).

26 J. H. Simpson, and H. Y. Carr, Phys. Rev. 111, 1201 (1958).

27 F. Mallamace, Proc. Natl. Acad. Sci. USA 106, 15097 (2009).

28 O. Mishima, Nature 384, 546 (1996).

29 O. Mishima, L. D. Calvert, and E. Whalley, Nature 314, 76 (1985).

30 O. Mishima, L. D. Calvert, and E. Whalley, Nature 310, 393 (1984).

31 H. E. Stanley, P. Kumar, G. Franzese, L. Xu, Z. Yan, M. G. Mazza, S. V. Buldyrev, S. H. Chen, and F. Mallamace, Eur. Phys. J. Spec. Top. 161, 1 (2008)

32 A. Geiger, and H. E. Stanley, Phys. Rev. Lett. 49, 1749 (1982).

33 H. Shintani, and H. Tanaka, Nat. Mater. 7, 870 (2008).

34 L. Xu, P. Kumar, S. V. Buldyrev, S. H. Chen, P. H. Poole, F. Sciortino, and H. E. Stanley, Proc. Natl. Acad. Sci. USA 102, 16558 (2005).

35 S.-H. Chen, F. Mallamace, C. Y. Mou, M. Broccio, C. Corsaro, A. Faraone, and L. Liu, Proc. Natl. Acad. Sci. USA, 103, 12974 (2006).

36 F. Mallamace, M. Broccio, C. Corsaro, A. Faraone, D. Majolino, V. Venuti, L. Liu, C. Y. Mou, and S. H. Chen, Proc. Natl. Acad. Sci. USA 104, 424 (2007).

37 S. Cerveny, F. Mallamace, J. Swenson, M. Vogel, and L. Xu, Chem. Rev. 116, 7608 (2016).

38 L. Xu, F. Mallamace, Z. Yan, F. W. Starr, S. V. Buldyrev, and H. Eugene Stanley, Nat. Phys. 5, 565 (2009).

39 C. Andreani, R. Senesi, M. Krzystyniak, G. Romanelli, and F. Fernandez-Alonso, Atomic quantum dynamics in materials research, in: Neutron Scattering-Applications in Biology, Chemistry, and Materials Science, edited by F. Fernandez-Alonso, and D. L. Price, (Academic Press, New York, 2017), pp. 403-457.
40 R. Senesi, G. Romanelli, M. A. Adams, and C. Andreani, Chem. Phys. 427, 111 (2013).

41 A. Parmentier, C. Andreani, G. Romanelli, J. J. Shephard, C. G. Salzmann, and R. Senesi, Front. Phys. 13, 136101 (2018), arXiv: 1709.07648 .

42 D. Flammini, A. Pietropaolo, R. Senesi, C. Andreani, F. McBride, A. Hodgson, M. A. Adams, L. Lin, and R. Car, J. Chem. Phys. 136, 024504 (2012).

43 G. Romanelli, M. Ceriotti, D. E. Manolopoulos, C. Pantalei, R. Senesi, and C. Andreani, J. Phys. Chem. Lett. 4, 3251 (2013).

44 G. Romanelli, A. Liscio, R. Senesi, R. Zamboni, E. Treossi, F. Liscio, G. Giambastiani, V. Palermo, F. Fernandez-Alonso, and C. Andreani, Carbon 108, 199 (2016).

45 G. Romanelli, R. Senesi, X. Zhang, K. P. Loh, and C. Andreani, Phys. Chem. Chem. Phys. 17, 31680 (2015).

46 A. H. Romero, P. L. Silvestrelli, and M. Parrinello, J. Chem. Phys. 115, 115 (2001).

47 F. Bloch, Phys. Rev. 70, 460 (1946).

48 E. M. Purcell, H. C. Torrey, and R. V. Pound, Phys. Rev. 69, 37 (1946).

49 N. Bloembergen, E. M. Purcell, and R. V. Pound, Phys. Rev. 73, 679 (1948).

50 C. Andreani, R. Senesi, F. Mallamace, M. Krzystyniak, L. Arcidiacono, STFC ISIS Neutron Muon Source, (2016), doi: 10.5286/ISIS.E.79114496.

51 G. Romanelli, M. Krzystyniak, R. Senesi, D. Raspino, J. Boxall, D. Pooley, S. Moorby, E. Schooneveld, N. J. Rhodes, C. Andreani, and F. Fernandez-Alonso, Meas. Sci. Technol. 28, 095501 (2017).

52 G. Romanelli, G. Festa, M. Krzystyniak, C. Andreani, F. FernandezAlonso, and R. Senesi, J. Phys.-Conf. Ser. 1055, 011001 (2018).

53 G. Romanelli, and M. Krzystyniak, Nucl. Instrum. Methods Phys. Res. Sect. A 819, 84 (2016).

54 C. Andreani, G. Romanelli, and R. Senesi, J. Phys. Chem. Lett. 7, 2216 (2016).

55 G. B. West, Phys. Rep. 18, 263 (1975).

56 G. F. Reiter, J. Mayers, and J. Noreland, Phys. Rev. B 65, 104305 (2002).

57 A. Pietropaolo, R. Senesi, C. Andreani, A. Botti, M. A. Ricci, and F. Bruni, Phys. Rev. Lett. 100, 127802 (2008).

58 Y. Finkelstein, and R. Moreh, Chem. Phys. 431-432, 58 (2014).

59 A. Parmentier, J. J. Shephard, G. Romanelli, R. Senesi, C. G. Salzmann, and C. Andreani, J. Phys. Chem. Lett. 6, 2038 (2015).

60 F. Mallamace, C. Corsaro, M. Broccio, C. Branca, N. GonzalezSegredo, J. Spooren, S. H. Chen, and H. E. Stanley, Proc. Natl. Acad. Sci. USA 105, 12725 (2008).

61 N. Matubayasi, C. Wakai, and M. Nakahara, Phys. Rev. Lett. 78, 2573 (1997).

62 B. G. Pfrommer, F. Mauri, and S. G. Louie, J. Am. Chem. Soc. 122, $123(2000)$

63 J. C. Hindman, J. Chem. Phys. 44, 4582 (1966).

64 A. Abragram, The Principles of Nuclear Magnetism (Clarendon, Oxford, 1961).

65 D. M. Grant, Encyclopedia of Nuclear Magnetic Resonance (Wiley, Chichester, 1996). p. 1298.

66 K. Modig, and B. Halle, J. Am. Chem. Soc. 124, 12031 (2002).

67 R. Ditchfield, J. Chem. Phys. 65, 3123 (1976).

68 B. Cheng, J. Behler, and M. Ceriotti, J. Phys. Chem. Lett. 7, 2210 (2016).

69 K. Nygärd, M. Hakala, T. Pylkkänen, S. Manninen, T. Buslaps, M. Itou, A. Andrejczuk, Y. Sakurai, M. Odelius, and K. Hämäläinen, J. Chem. Phys. 126, 154508 (2007).

70 C. A. Angell, J. Shuppert, and J. C. Tucker, J. Phys. Chem. 77, 3092 (1973).

71 D. R. Kinney, I. S. Chuang, and G. E. Maciel, J. Am. Chem. Soc. 115, 6786 (1993).

72 R. Senesi, D. Flammini, A. I. Kolesnikov, D. Murray, G. Galli, and C. 
Andreani, J. Chem. Phys. 139, 074504 (2013).

73 C. Pantalei, A. Pietropaolo, R. Senesi, S. Imberti, C. Andreani, J. Mayers, C. Burnham, and G. Reiter, Phys. Rev. Lett. 100, 177801 (2008).

74 A. K. Soper, Water and ice structure in the range 220-365 $\mathrm{K}$ from radiation total scattering experiments, in: Water: Fundamentals as the Basis for Understanding the Environment and Promoting Technology (IOS Press, Amsterdam, 2015), pp. 151-172.

75 K. Amann-Winkel, M. C. Bellissent-Funel, L. E. Bove, T. Loerting, A. Nilsson, A. Paciaroni, D. Schlesinger, and L. Skinner, Chem. Rev. 116, 7570 (2016).

76 A. Pietropaolo, R. Senesi, C. Andreani, and J. Mayers, Braz. J. Phys. 39, 318 (2009).

77 C. Andreani, G. Romanelli, and R. Senesi, Chem. Phys. 427, 106
(2013).

78 Y. Huang, X. Zhang, Z. Ma, W. Li, Y. Zhou, J. Zhou, W. Zheng, and C. Q. Sun, Sci. Rep. 3, 3005 (2013).

79 N. Galamba, J. Phys.-Condens. Matter 29, 015101 (2017).

80 E. B. Moore, and V. Molinero, J. Chem. Phys. 130, 244505 (2009).

81 D. Majolino, F. Mallamace, P. Migliardo, F. Aliotta, N. Micali, and C. Vasi, Phys. Rev. E 47, 2669 (1993).

82 J. A. Sellberg, C. Huang, T. A. McQueen, N. D. Loh, H. Laksmono, D. Schlesinger, R. G. Sierra, D. Nordlund, C. Y. Hampton, D. Starodub, D. P. DePonte, M. Beye, C. Chen, A. V. Martin, A. Barty, K. T. Wikfeldt, T. M. Weiss, C. Caronna, J. Feldkamp, L. B. Skinner, M. M. Seibert, M. Messerschmidt, G. J. Williams, S. Boutet, L. G. M. Pettersson, M. J. Bogan, and A. Nilsson, Nature 510, 381 (2014). 